\title{
Hybrid Beamformers Design for MIMO Relay Networks in Millimeter Wave
}

DOI:

10.1109/WCNC.2017.7925884

\section{Document Version}

Accepted author manuscript

Link to publication record in Manchester Research Explorer

\section{Citation for published version (APA):}

Abbas, H., \& Hamdi, K. (2017). Hybrid Beamformers Design for MIMO Relay Networks in Millimeter Wave. In 2017 IEEE Wireless Communications and Networking Conference IEEE. https://doi.org/10.1109/WCNC.2017.7925884

\section{Published in:}

2017 IEEE Wireless Communications and Networking Conference

\section{Citing this paper}

Please note that where the full-text provided on Manchester Research Explorer is the Author Accepted Manuscript or Proof version this may differ from the final Published version. If citing, it is advised that you check and use the publisher's definitive version.

\section{General rights}

Copyright and moral rights for the publications made accessible in the Research Explorer are retained by the authors and/or other copyright owners and it is a condition of accessing publications that users recognise and abide by the legal requirements associated with these rights.

\section{Takedown policy}

If you believe that this document breaches copyright please refer to the University of Manchester's Takedown Procedures [http://man.ac.uk/04Y6Bo] or contact uml.scholarlycommunications@manchester.ac.uk providing relevant details, so we can investigate your claim.

\section{OPEN ACCESS}




\title{
Hybrid Beamformers Design for MIMO Relay Networks in Millimeter Wave
}

\author{
Hatem Abbas, and Khairi Hamdi, \\ School of Electrical and Electronic Engineering, \\ The University of Manchester, Manchester, UK. \\ emails: hatem.h.abbas@ieee.org, k.hamdi@manchester.ac.uk
}

\begin{abstract}
One of the challenges for the wireless networks is meeting the high demand for cellular data. One approach is to utilize the wide bandwidth provided by the millimeter wave (mmWave) frequencies. In this work, we propose using multiple relays to link two points in the outdoor mmWave environments. We use hybrid (analog and digital) beamformers in the Amplify-and-Forward (AF) half duplex relays to overcome the propagation losses usually arise in such environments. The design is based on the the widely known Orthogonal Match Pursuit (OMP) algorithm. Results show significant performance improvement for multiple relay links as compared to links with single relay. The results also discuss the influence of the array size and the number of RF chains on the overall performance.

Index Terms-Millimeter wave, ABF, Relay Networks, Hybrid beamforming, Multi-Relay, 5G.
\end{abstract}

\section{INTRODUCTION}

The urge for higher data rates is increasing with the development of the smart wireless devices [1]. In order for the next generation cellular networks to meet these demands, three scenarios are proposed: more frequency re-usability, enhanced spectral efficiency, and higher frequency bandwidth [2], [3]. With the relatively wide spectrum and the cost effective CMOS technology, mmWave is anticipated to be the core of the future wireless networks [4].

In spite of its benefits, $60 \mathrm{GHz}$ frequencies in the outdoors environment endure relatively high propagation losses. Recently, research has been proposing solutions to overcome the losses such as higher directivity gain using beamforming, smaller cells, and cooperative networks [5]-[8].

Increasing the directivity of the signal is considered feasible in mmWave systems due to the large antenna arrays available as a result of the short wavelength [9]. However, with the complexity of the digital beamformers (DBF) and the limitations of the analog beamformers (ABF), hybrid beamformers (HBF) have been widely adapted as the most efficient solution for signal directivity in mmWave systems [10], [11]. The number of the RF chains in hybrid systems is less than the number of antennas which makes hybrid systems cost efficient [9].

Non-iterative joint design for the $\mathrm{Tx} / \mathrm{Rx}$ analog processors at the $60 \mathrm{GHz}$ band was introduced in [12], [13]. The joint design of the analog and the digital processors by utilizing the sparsity of the mmWave channel was introduced in [10], [14]. The design assumes full channel state information (CSI) and used iterations of OMP based algorithm [15]. This method gained importance due to the low complexity of the design.
The short coverage of the mmWave systems and the flexibility provided by relay networks make them perfect match to be key elements in the future of communications. The study of twohop multiple single-antenna relay networks was introduced in [16], and the optimization of the MIMO relay networks has been investigated in [17]. However, In millimeter waves, there is a shortage of research in the design of single relay networks, rather than multiple relays. In a recent study, half duplex (HD) relay network was introduced in mmWave environment where the sparsity principle is used to jointly design the hybrid beamforming processors at the relay [18]. Yet, multiple relays network have not been studied throughly.

In this work, we propose using multiple relays to link two points over the mmWave frequencies. The sparsity approach for designing the hybrid processors [10], [19] is utilized to design the analog and digital matrices of the relays processors. We study the performance of the proposed algorithm under different assumptions and real life scenarios. The main contribution of this work is revising the OMP principle to be used in multiple relays network which yields a modified algorithm that takes in consideration the relay network limitations.

The rest of the paper organized as follows. The system and channel models with the main assumptions used in the paper are presented in Section II. The design of the hybrid processors using the sparsity algorithm is presented in Section III. Simulation results are presented in Section IV before concluding the paper in Section V.

We use the following notation throughout this paper: A is a matrix; $\mathbf{a}$ is a vector; $a$ is a scalar. $\mathbf{A}^{(i)}$ is the $i^{\text {th }}$ column of $\mathbf{A}, \mathbf{A}_{(i, j)}$ is the $(i, j)$-th entry of $\mathbf{A} .\|\mathbf{A}\|_{F}$ is the Frobenius norm of $\mathbf{A}$, whereas $(\mathbf{A})^{T},(\mathbf{A})^{H},(\mathbf{A})^{-1}$ denote transpose, Hermitian, and inverse respectively. $\|\mathbf{a}\|_{p}$ is the $p$ norm of $\mathbf{a},[\mathbf{A} \mid \mathbf{B}]$ denotes horizontal concatenation. $\mathbf{I}_{N}$ is the $N \times N$ identity matrix; $\mathbf{0}_{M \times N}$ is the $M \times N$ all-zeros matrix. $\mathcal{C N}(\mathrm{a} ; \mathbf{A})$ is a complex Gaussian vector with mean a and covariance matrix A. \# is used to indicate (number of). bd[.] means block-diagonal matrix.

\section{System And Channel Models}

We propose a multiple relay network where the source communicates with the destination through multiple relays. The destination and each of the relays are equipped with HBF system to direct the signal towards the preferred direction. 


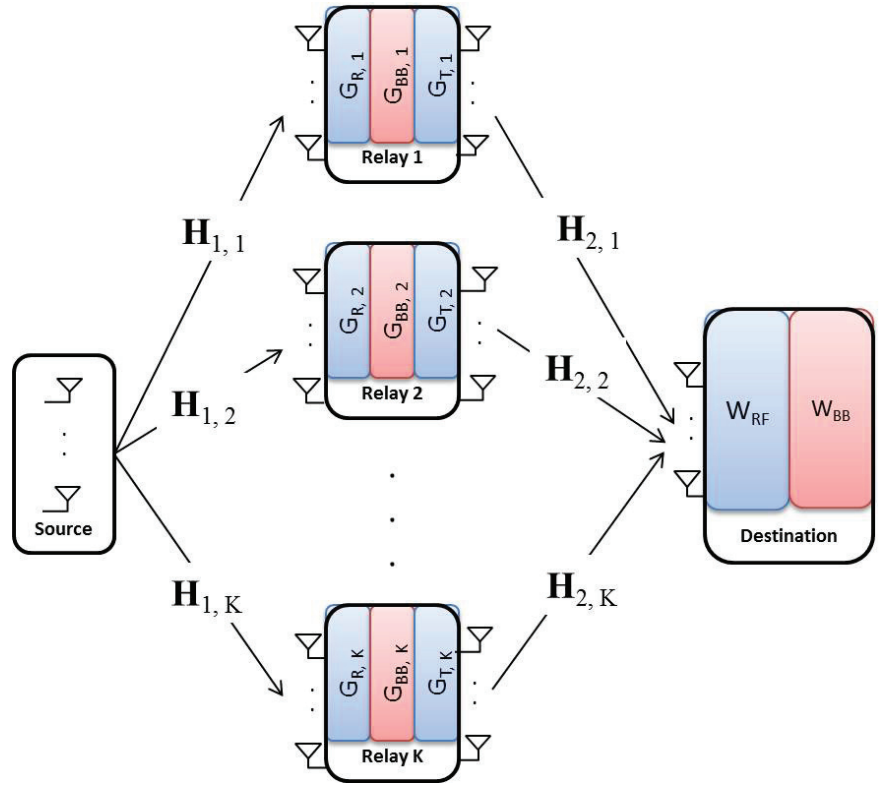

Fig. 1: Multi-relay system with hybrid beamforming

\section{A. System Model}

As illustrated in Fig. 1, single source is communicating with a single destination through $K$ relays. The source is equipped with $N_{\mathrm{T}}$ antennas, the destination has $N_{\mathrm{R}}$ antennas, and each relay has $M_{\mathrm{R}} / M_{\mathrm{T}}$ receive/transmit antennas. Amplify-andForward relay scheme is used with two time slots to perform the process. During the first time slot, the source transmits the $N_{T} \times 1$ signal s with $\mathbb{E}\left[\mathbf{s s}^{H}\right]=\rho \mathbf{I}$. The $k$ th relay received signal will be

$$
\mathbf{y}_{r, k}=\mathbf{H}_{1, k} \mathbf{s}+\mathbf{n}_{1, k}
$$

where $\mathbf{H}_{1, k}$ is the mmWave MIMO channel between the source and the $k$ th relay, $\mathbf{n}_{1, k}$ is the additive noise vector with covariance matrix $\mathcal{R}_{n_{1, k}}=\sigma_{n_{1, k}}^{2} \mathbf{I}_{M_{\mathrm{R}}}$. During the second time slot, the relays transmit their signals to the destination. The transmitted signal of the $k$ th relay can be written as

$$
\mathbf{x}_{r, k}=\mathbf{G}_{T, k} \mathbf{G}_{B B, k} \mathbf{G}_{R, k}^{H} \mathbf{y}_{r, k}
$$

where $\mathbf{G}_{T, k}, \mathbf{G}_{B B, k}, \mathbf{G}_{R, k}$ are the $M_{\mathrm{T}} \times M_{\mathrm{RF}}$ analog transmit matrix, the $M_{\mathrm{RF}} \times M_{\mathrm{RF}}$ baseband matrix, and the $M_{\mathrm{R}} \times M_{\mathrm{RF}}$ analog receive matrix of the hybrid beamformer of the $k$ th relay, respectively. $M_{\mathrm{RF}}$ is the number of $\mathrm{RF}$ chains at each side of the relay. $\mathbf{G}_{T, k}, \mathbf{G}_{R, k}$ are implemented using analog phase shifters, therefore, their entries have equal norm, i.e. for the transmit side $\left(\mathbf{G}_{T, k}^{(i)} \mathbf{G}_{T, k}^{(i) H}\right)_{(\ell, \ell)}=M_{\mathrm{T}}^{-1}$, while $\mathbf{G}_{B B, k}$ has no hardware constraints except the total power constraint. We also define the ratio $\beta=\frac{\# \text { ant }}{\# \text { RFchains }}$ to be the hybridization ratio. $1 \leq \beta \leq \#$ antennas, with $\beta=1$ being a fully digital system, and $\beta=$ \#antennas being an analog beamformer.

Now, defining $\mathbf{G}_{k}=\mathbf{G}_{T, k} \mathbf{G}_{B B, k} \mathbf{G}_{R, k}^{H}$, the destination received signal can be expressed as

$$
\mathbf{y}=\sum_{k=1}^{K}\left(\mathbf{H}_{2, k} \mathbf{G}_{k} \mathbf{H}_{1, k} \mathbf{s}+\mathbf{G}_{k} \mathbf{n}_{1, k}\right)+\mathbf{n}_{2}
$$

where $\mathbf{H}_{2, k}$ is the mmWave channel between the $k$ th relay and the destination, and $\mathbf{n}_{2}$ is the additive white noise at the destination with covariance matrix $\mathcal{R}_{n_{2}}=\sigma_{n_{2}}^{2} \mathbf{I}_{N_{\mathrm{R}}}$. The destination receives the signal through $N_{\mathrm{R}} \times N_{\mathrm{RF}} \mathrm{RF}$ combiner $\mathrm{W}_{\mathrm{RF}}$, and processes it using the $N_{\mathrm{RF}} \times N_{\mathrm{S}}$ baseband matrix $\mathbf{W}_{\mathrm{BB}}$, the processed signal is given by

$$
\hat{\mathbf{s}}=\mathbf{W}^{H} \mathbf{H}_{2} \mathbf{G} \mathbf{H}_{1} \mathbf{s}+\mathbf{W}^{H} \mathbf{G n}_{1}+\mathbf{W}^{H} \mathbf{n}_{2}
$$

where $N_{\mathrm{RF}}$ is the number of RF chains at the destination, $\mathbf{W}=\mathbf{W}_{\mathrm{RF}} \mathbf{W}_{\mathrm{BB}}, \mathbf{H}_{1}=\left[\mathbf{H}_{1,1}^{T}, \mathbf{H}_{1,2}^{T}, \cdots, \mathbf{H}_{1, K}^{T}\right]^{T}$ is the $K M_{\mathrm{R}} \times N_{\mathrm{T}}$ channel matrix between the source and the relays, $\mathbf{n}_{1}=\left[\mathbf{n}_{1,1}^{T}, \mathbf{n}_{1,2}^{T}, \cdots, \mathbf{n}_{1, K}^{T}\right]^{T}$ is the $K M_{\mathrm{R}} \times 1$ noise matrix at the relays, $\mathbf{G}=\operatorname{bd}\left[\mathbf{G}_{1}, \mathbf{G}_{2}, \cdots, \mathbf{G}_{K}\right]$ is the $K M_{\mathrm{T}} \times K M_{\mathrm{R}}$ equivalent block diagonal relay amplifying matrix, and $\mathbf{H}_{2}=$ $\left[\mathbf{H}_{2,1}, \mathbf{H}_{2,2}, \cdots, \mathbf{H}_{2, K}\right]$ is the $M_{\mathrm{R}} \times K M_{\mathrm{T}}$ channel matrix between the all the relay nodes and the destination.

\section{B. Channel Model}

The measurements of outdoor mmWave $28 \mathrm{GHz}$ and the $72 \mathrm{GHz}$ channels showed that they normally have limited scattering [4], [20] where the multipath components are mainly generated by reflection. To incorporate this effect, we adopt a geometric channel model based on the extended SalehValenzuela model in which the channel is assumed to be a sum of $L$ scattering clusters, each of which contributes $P$ propagation paths to the channel [21], [22]. With half-wave spaced ULAs used at the transmitter and the receiver, the channel matrix can be expressed as

$$
\mathbf{H}_{q, k}=\frac{1}{\sqrt{L_{q} P_{q}}} \sum_{\ell=1}^{L_{q}} \sum_{p=1}^{P_{q}} \alpha_{p \ell}^{q, k} \mathbf{a}_{r}\left(\theta_{p \ell}^{q, k}\right) \mathbf{a}_{t}^{H}\left(\phi_{p \ell}^{q, k}\right),
$$

where $q \in\{1,2\}, k \in\{1, \cdots, K\}, \alpha_{p \ell}$ is the complex smallscale fading gain of the $p$-th sub-path of the $\ell$-th cluster. $\theta_{p \ell}\left(\phi_{p \ell}\right)$ is the azimuth angle of arrival (angle of departure). The gains $\alpha_{p \ell}$ are complex Gaussian random variables with zero mean and variance $\sigma_{\alpha}^{2}$. The mean angle associated with each cluster is uniformly distributed over $[-\pi, \pi]$, and the distribution of the difference between an AoA $\{\mathrm{AoD}\}$ and its mean is Laplacian with angular standard deviation $\gamma$. The vectors $\mathbf{a}_{r}\left(\theta_{p \ell}^{r}\right)$ and $\mathbf{a}_{t}^{H}\left(\theta_{p \ell}^{t}\right)$ are the array response vectors at the receiver and transmitter, respectively.

Without loss of generality, we are using uniform linear array (ULA) to get the results in the simulation in Section IV. In this case, the array response vector (a) is defined as

$$
\mathbf{a}(\theta)=\frac{1}{\sqrt{M}}\left[1 e^{j 2 \pi \frac{d}{\lambda} \sin (\theta)} \cdots e^{j 2 \pi(M-1) \frac{d}{\lambda} \sin (\theta)}\right]^{T}
$$

where $d$ is the space between the antennas, $M$ is the number of antennas, and $\lambda$ is the wavelength. 


$$
\begin{array}{r}
\left(\mathbf{G}_{k}^{o}, \mathbf{W}^{\circ}\right)=\underset{\mathbf{G}_{\mathrm{R}, \mathrm{k}}, \mathbf{G}_{\mathrm{BB}, \mathrm{k}}, \mathbf{G}_{\mathrm{T}, \mathrm{k}}, \mathbf{W}_{\mathrm{RF}}, \mathbf{W}_{\mathrm{BB}}}{\operatorname{argmin}}\left\|\mathbf{s}-\mathbf{W}_{\mathrm{BB}}^{H} \mathbf{W}_{\mathrm{RF}}^{H} \mathbf{y}\right\|_{F}, \\
\text { s.t. } \mathbf{G}_{\mathrm{T}, \mathrm{k}} \in \mathcal{G}_{\mathrm{RF}}^{t, k}, \mathbf{G}_{\mathrm{R}, \mathrm{k}} \in \mathcal{G}_{\mathrm{RF}}^{r, k}, \mathbf{W}_{\mathrm{RF}} \in \mathcal{W}_{\mathrm{RF}} \\
\left\|\mathbf{G}_{T, k} \mathbf{G}_{B B, k} \mathbf{G}_{R, k}^{H}\left(\mathbf{H}_{1, k} \mathbf{s}+\mathbf{n}_{1, k}\right)\right\|_{F}^{2}=\rho_{k}
\end{array}
$$

\section{HYBRID BEAMFORMER DESIGN}

The hybrid beamformer at the relay consists of one analog beamformer at the receive side, a digital baseband processor, and another analog beamformer at the transmitter side of each relay. Similarly, the hybrid processor at the destination consists of an analog processor and a digital baseband matrix.

The design of the hybrid processors that minimize the error between the estimated signal and the transmitted signal requires a joint design for all the matrices in the system, as described in (7)-(9), at the top of the page. $\mathcal{G}_{\mathrm{RF}}^{t, k}, \mathcal{G}_{\mathrm{RF}}^{r, k}$, and $\mathcal{W}_{\mathrm{RF}}$ in (8) represent the sets of the feasible RF processors at the relay and the destination. While (9) describe the power constraint with $\rho_{k}$ is the transmitted power of the $k$ th relay.

Jointly optimizing the problem in (7) with the constrains in (8) is known to be intractable [10], [23]. A near optimal solution is suggested by firstly decoupling the relay and destination, then minimize the Frobenius norm of the difference between the hybrid processors and the unconstrained processors $\mathbf{G}_{u, k}, \mathbf{W}_{\mathrm{u}}$. The unconstrained processors are designed with fully complex $\mathrm{RF}$ chains is described in Appendix A.

Now, suppose the feasible RF sets are given, the hybrid beamformer could be designed based on the sparse approximation. For instance, in order to formulate the design problem at the $k$ th relay, we define $M_{T} \times N$ matrix $\mathbf{B}_{\mathrm{T}, \mathrm{k}}=\left[\mathbf{A}_{T_{2, k}} \mid \mathbf{A}\right]$ (with $N>P \times L$ ), where $\mathbf{A}_{T_{2, k}}$ columns consist of the transmitter array response vectors of the relay-to-destination channel, i.e. $\mathbf{A}_{T_{2, k}}=\left[\mathbf{a}_{t}\left(\theta_{1}^{2, k}\right) \mathbf{a}_{t}\left(\theta_{2}^{2, k}\right) \cdots \mathbf{a}_{t}\left(\theta_{P \times L}^{2, k}\right)\right] . \mathbf{A}$ is an optional matrix whose columns are chosen to satisfy arbitrary analog beamforming constraints and to be used when $P \times L \leq N_{\mathrm{RF}}$. Similarly, we define $\mathbf{B}_{\mathrm{R}, \mathrm{k}}=\left[\mathbf{A}_{R_{1, k}} \mid \mathbf{A}\right]$, with $\mathbf{A}_{R_{1, k}}=\left[\mathbf{a}_{r}\left(\phi_{1}^{1, k}\right) \mathbf{a}_{r}\left(\phi_{2}^{1, k}\right) \cdots \mathbf{a}_{r}\left(\phi_{P \times L}^{1, k}\right)\right]$. Then, we write the problem of minimizing the error between the hybrid beamformer and the unconstrained precoder as

$$
\begin{array}{r}
\tilde{\mathbf{G}}_{\mathrm{BB}, \mathrm{k}}^{\mathrm{o}}=\underset{\tilde{\mathbf{G}}_{\mathrm{BB}, \mathrm{k}}}{\operatorname{argmin}}\left\|\mathbf{G}_{\mathrm{u}, \mathrm{k}}-\mathbf{B}_{\mathrm{T}, \mathrm{k}} \tilde{\mathbf{G}}_{\mathrm{BB}, \mathrm{k}} \mathbf{B}_{\mathrm{R}, \mathrm{k}}^{H}\right\|_{F}, \\
\text { s.t. } \quad\left\|\operatorname{diag}\left(\tilde{\mathbf{G}}_{\mathrm{BB}, \mathrm{k}} \tilde{\mathbf{G}}_{\mathrm{BB}, \mathrm{k}}^{H}\right)\right\|_{0}=M_{\mathrm{RF}}, \\
\\
\quad\left\|\mathbf{B}_{\mathrm{T}, \mathrm{k}} \tilde{\mathbf{G}}_{\mathrm{BB}, \mathrm{k}} \mathbf{B}_{\mathrm{R}, \mathrm{k}}^{H}\right\|_{F}^{2}=\rho_{k}
\end{array}
$$

where $\tilde{\mathbf{G}}_{\mathrm{BB}, \mathrm{k}}$ is an $N \times N_{S}$ sparse matrix having $M_{\mathrm{RF}}$ nonzero rows (which constitute of $\mathbf{G}_{\mathrm{BB}, \mathrm{k}}$ ), $\left\|\operatorname{diag}\left(\tilde{\mathbf{G}}_{\mathrm{BB}, \mathrm{k}} \tilde{\mathbf{G}}_{\mathrm{BB}, \mathrm{k}}^{H}\right)\right\|_{0}=$ $M_{\mathrm{RF}}$ ensures that $\tilde{\mathbf{G}}_{\mathrm{BB}, \mathrm{k}}$ is $M_{\mathrm{RF}}$ sparse. As only $M_{\mathrm{RF}}$ rows of $\tilde{\mathbf{G}}_{\mathrm{BB}, \mathrm{k}}$ are nonzero, only $M_{\mathrm{RF}}$ vectors of $\mathbf{B}_{\mathrm{T}, \mathrm{k}}$ and $\mathbf{B}_{\mathrm{R}, \mathrm{k}}$ are selected, and they represent the vectors of $\mathrm{G}_{\mathrm{T}, \mathrm{k}}^{\circ}$ and $\mathrm{G}_{\mathrm{R}, \mathrm{k}}^{\mathrm{o}}$, respectively. In other words, in (10), the columns of $\mathbf{G}_{\mathrm{u}, \mathrm{k}}$ are approximated by a linear combination of $\mathbf{B}_{\mathrm{T}, \mathrm{k}}$ and $\mathbf{B}_{\mathrm{R}, \mathrm{k}}$ with the nonzero elements of $\tilde{\mathbf{G}}_{\mathrm{BB}, \mathrm{k}}$ specify the weights.

An OMP algorithm for jointly designing $\mathbf{G}_{\mathrm{T}, \mathrm{k}}^{\mathrm{o}}, \mathbf{G}_{\mathrm{R}, \mathrm{k}}^{\mathrm{o}}$ and the baseband processor $\mathbf{G}_{\mathrm{BB}, \mathrm{k}}^{\mathrm{o}}$ is outlined in Algorithm 1. During the first iteration, the columns of $\mathbf{B}_{\mathrm{T}, \mathrm{k}}$ and $\mathbf{B}_{\mathrm{R}, \mathrm{k}}$ that correlate the most with $\mathbf{G}_{\mathrm{u}, \mathrm{k}}$ are identified (step 1a), then added to $\mathbf{G}_{\mathrm{T}, \mathrm{k}}$ and $\mathbf{G}_{\mathrm{R}, \mathrm{k}}$, respectively (step $1 b$ ). $\mathbf{G}_{\mathrm{BB}, \mathrm{k}}$ is obtained by solving the least squares problem $\left(\mathbf{G}_{\mathrm{u}, \mathrm{k}}=\mathbf{G}_{\mathrm{T}, \mathrm{k}} \mathbf{G}_{\mathrm{BB}, \mathrm{k}} \mathbf{G}_{\mathrm{R}, \mathrm{k}}^{H}\right)$, (step 1c). The residual is updated by subtracting the selected columns (step 1d). On each of the successive iterations, the columns of $\mathbf{B}_{\mathrm{T}, \mathrm{k}}$ and $\mathbf{B}_{\mathrm{R}, \mathrm{k}}$ that correlate the most with the residual $\mathbf{G}_{r e s, k}$ and $\mathbf{G}_{\mathrm{R}, \mathrm{k}}$ or $\mathbf{G}_{\mathrm{T}, \mathrm{k}}$ are identified (step 2) and then added to the RF beamformers. Similar algorithms could be used to design the RF processors at the destination.

\section{Simulation Results}

The performance of the proposed algorithm is examined by measuring the achievable rate through computer simulations. All the relays are assumed to have the same distance from the source and destination so that all the channels have the same statistics. Each of the propagation channels is modeled with $L$ clusters channel and each cluster contributes with single ray with uniformly random AoAs and AoDs. The complex path gains are assumed to be Gaussian distributed with equal variances. We assume a ULA with inter-elements spacing $d=\lambda / 2$. The noise variance $\sigma_{n_{1, k}}^{2}=\sigma_{n_{2}}^{2}$, and the signal to noise ratio is defined as $S N R=\frac{\rho}{N_{S} \sigma^{2}}$. Other simulation parameters as follows: the number of $\mathrm{RF}$ chains $N_{\mathrm{RF}}, M_{\mathrm{RF}} \in\{3,4,5\}$, and the number of data streams equals the number of antennas at the source.

Fig. 2 compares the hybrid system performance with the unconstrained system in [17] and the single relay system. The results show an improved performance with the addition of relays to the system. The results also indicate that the unconstrained processors outperform the hybrid processor system. It requires using 4 hybrid relays to get the performance of a single unconstrained relay system. The effect of the number of the relays is further examined in Fig. 3. The results are obtained using channels with $L=20$ paths and array size of 6 antennas at the source/destination, and 8 antennas at the relay receive/transmit sides.

Finally, the system performance was examined for different array sizes at each of the relays for fixed source/destination arrays as shown in Fig. 4. The number of antennas at the relays could be adjusted to reach the peak performance. For a fixed number of RF chains, when the array size increases the value of $\beta$ increases from about 1 to higher values, and the system becomes closer to analog than digital. Increasing the number 
$\overline{\text { Algorithm } 1 \text { Orthogonal Match Pursuit based algorithm to }}$ design the relay hybrid processors.

\section{Input:}

- Define the analog sets:

$\mathbf{B}_{\mathrm{T}, \mathrm{k}}=\left[\mathbf{A}_{T_{2, k}} \mid \mathbf{A}\right], \mathbf{B}_{\mathrm{R}, \mathrm{k}}=\left[\mathbf{A}_{R_{1, k}} \mid \mathbf{A}\right]$

- Define the unconstrained processors $\mathbf{G}_{\mathrm{u}, \mathrm{k}}$

Output: $\mathbf{G}_{\mathrm{T}, \mathrm{k}}, \mathbf{G}_{\mathrm{BB}, \mathrm{k}}, \mathbf{G}_{\mathrm{R}, \mathrm{k}}(1 \leq k \leq K)$

Initialization: Set up the initial residual matrix $\mathbf{G}_{\mathrm{res}, \mathrm{k}}=\mathbf{G}_{\mathrm{u}, \mathrm{k}}$

1) First Iteration $(i=1)$.

a) Identify the columns of $\mathbf{B}_{\mathrm{T}, \mathrm{k}}, \mathbf{B}_{\mathrm{R}, \mathrm{k}}$ that correlate the most with $\mathbf{G}_{\mathrm{u}, \mathrm{k}}$ :

$$
\begin{gathered}
\boldsymbol{\Psi}_{R}=\mathbf{G}_{\mathrm{u}, \mathrm{k}} \mathbf{B}_{\mathrm{R}, \mathrm{k}} \\
\boldsymbol{\Psi}_{T}=\mathbf{B}_{\mathrm{T}, \mathrm{k}}^{H} \mathbf{G}_{\mathrm{u}, \mathrm{k}} \\
k=\underset{n=1, \cdots, P \times L}{\operatorname{argmax}}\left(\boldsymbol{\Psi}_{R}^{H} \boldsymbol{\Psi}_{R}\right)_{n, n} \\
v=\underset{n=1, \cdots, P \times L}{\operatorname{argmax}}\left(\boldsymbol{\Psi}_{T} \boldsymbol{\Psi}_{T}^{H}\right)_{n, n}
\end{gathered}
$$

b) Add the identified columns to $\mathbf{G}_{\mathrm{T}, \mathrm{k}}, \mathbf{G}_{\mathrm{R}, \mathrm{k}}$ :

$$
\begin{aligned}
\mathbf{G}_{\mathrm{R}, \mathrm{k}}^{(i)} & =\mathbf{B}_{\mathrm{R}, \mathrm{k}}^{(k)} \\
\mathbf{G}_{\mathrm{T}, \mathrm{k}}^{(i)} & =\mathbf{B}_{\mathrm{T}, \mathrm{k}}^{(v)}
\end{aligned}
$$

c) Calculate $\mathbf{G}_{\mathrm{BB}, \mathrm{k}}$ by solving the least squares problem:

$$
\begin{aligned}
\mathbf{G}_{\mathrm{BB}, \mathrm{k}}= & \left(\mathbf{G}_{\mathrm{T}, \mathrm{k}}^{H} \mathbf{G}_{\mathrm{T}, \mathrm{k}}\right)^{-1} \mathbf{G}_{\mathrm{T}, \mathrm{k}}^{H} \mathbf{G}_{\mathrm{u}, \mathrm{k}} \mathbf{G}_{\mathrm{R}, \mathrm{k}} \\
& \left(\mathbf{G}_{\mathrm{R}, \mathrm{k}}^{H} \mathbf{G}_{\mathrm{R}, \mathrm{k}}\right)^{-1}
\end{aligned}
$$

d) Calculate the residual:

$$
\mathbf{G}_{\mathrm{res}, \mathrm{k}}=\frac{\mathbf{G}_{\mathrm{u}, \mathrm{k}}-\mathbf{G}_{\mathrm{T}, \mathrm{k}} \mathbf{G}_{\mathrm{BB}, \mathrm{k}} \mathbf{G}_{\mathrm{R}, \mathrm{k}}^{H}}{\left\|\mathbf{G}_{\mathrm{u}, \mathrm{k}}-\mathbf{G}_{\mathrm{T}, \mathrm{k}} \mathbf{G}_{\mathrm{BB}, \mathrm{k}} \mathbf{G}_{\mathrm{R}, \mathrm{k}}^{H}\right\|_{F}}
$$

2) The remaining $\left(M_{\mathrm{R}}-1\right)$ Iterations $\left(i=2, \cdots, M_{\mathrm{R}}\right)$.

a) Identify the columns of $\mathbf{B}_{\mathrm{T}, \mathrm{k}}, \mathbf{B}_{\mathrm{R}, \mathrm{k}}$ that correlate the most with the residual $\mathbf{G}_{\mathrm{res}, \mathrm{k}}$ :

$$
\begin{aligned}
\boldsymbol{\Psi}_{R} & =\mathbf{G}_{\mathrm{T}, \mathrm{k}}^{H} \mathbf{G}_{\mathrm{res}, \mathrm{k}} \mathbf{B}_{\mathrm{R}, \mathrm{k}} \\
\boldsymbol{\Psi}_{T} & =\mathbf{B}_{\mathrm{T}, \mathrm{k}}^{H} \mathbf{G}_{\mathrm{res}, \mathrm{k}} \mathbf{G}_{\mathrm{R}, \mathrm{k}}
\end{aligned}
$$

b) Repeat steps (1b) to (1d) above.

3) Satisfy the power condition:

$$
\left\|\mathbf{G}_{T, k} \mathbf{G}_{B B, k} \mathbf{G}_{R, k}^{H}\left(\mathbf{H}_{1, k} \mathbf{s}+\mathbf{n}_{1, k}\right)\right\|_{F}^{2}=\rho_{k}
$$

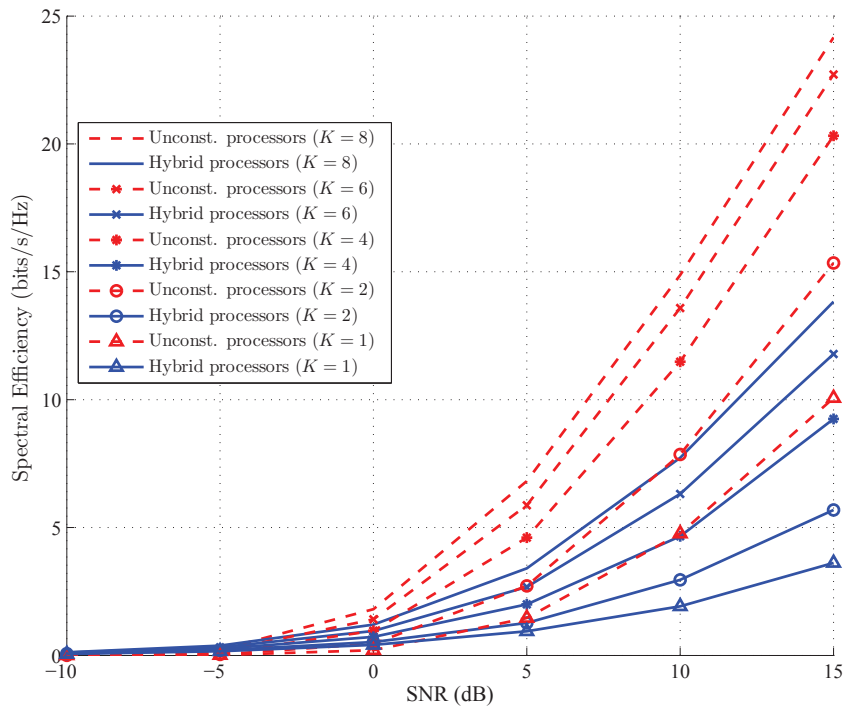

Fig. 2: Spectral efficiency versus SNR with $L=20, N_{R F} \in$ $\{3,4,5\}, N=20$, and $K=6$ relays. The array size of the source relay, and destination are $N_{T}=6, M_{R}=8, M_{T}=$ $8, N_{R}=6$.

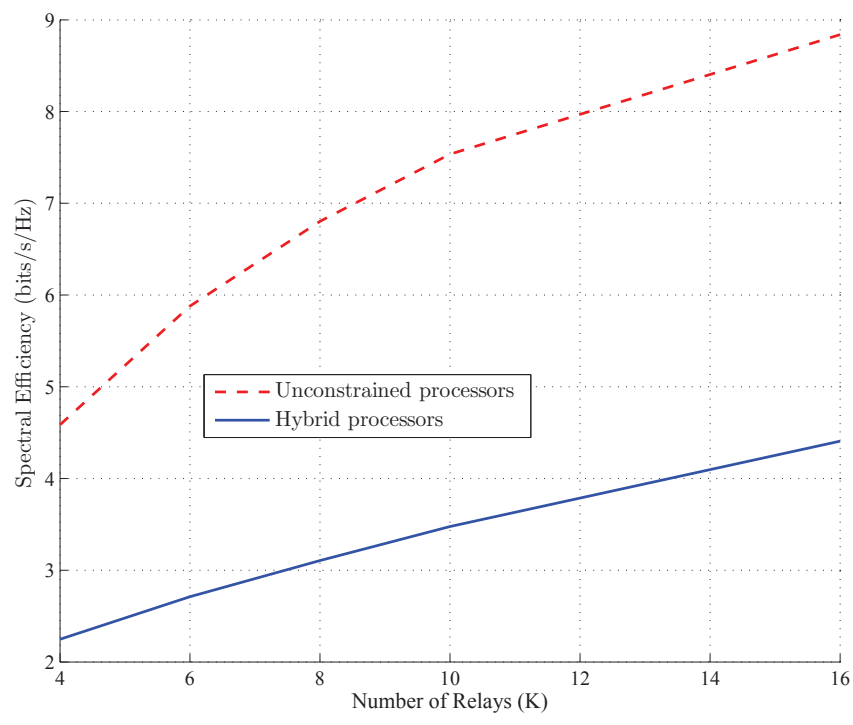

Fig. 3: Spectral efficiency versus number of relays when $L=$ $20, S N R=5 d B$, and $N_{R F}=4$. The array size of the source relay, and destination are $N_{T}=6, M_{R}=8, M_{T}=8, N_{R}=6$. 


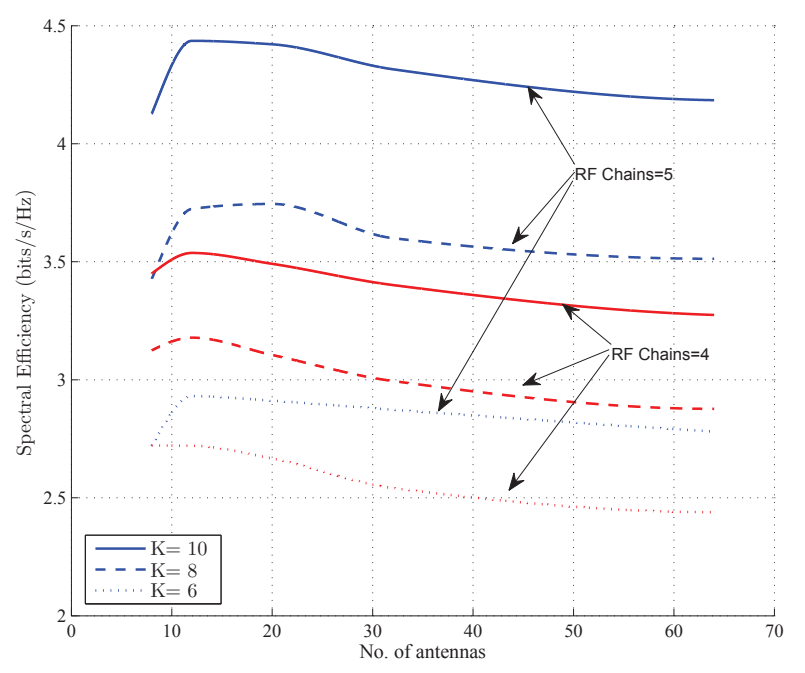

Fig. 4: Spectral efficiency versus array size at the relay transmit and receive sides. Number of antennas at the source and destination fixed to $N_{T}=N_{R}=6 . K \in\{6,8,10\}$, $M_{\mathrm{RF}}, N_{\mathrm{RF}} \in\{4,5\}, \mathrm{SNR}=5 \mathrm{~dB}$, and $L=20$.

of RF chains has a positive impact on the system performance, as it is clear from the results.

\section{Conclusion}

In this paper, we presented the two-hop multiple relays networks in millimeter waves. The relays and the destination are equipped with multiple antennas and hybrid beamformers to direct the signal and obtain higher beamforming gains to overcome the path losses in outdoors mmWave environments. Sparsity based algorithm for designing the hybrid (analog/digital) precoders was adapted to be used at the destination, and the AF relays. The results showed that adding more relays to the system will increase the system performance. The results indicate that the unconstrained processors outperforms the hybrid processors and it requires about 4 hybrid relays to deliver the performance of one unconstrained relay. Increasing the number of antennas at the relays have a positive impact on the performance. A similar impact is obtained by adding more RF chains to the system. MMSE is used to design the unconstrained processors at the relays and the destination with no processing at the source.

\section{APPENDIX A}

\section{UNCONSTRAINED PROCESSORS DESIGN}

The unconstrained precoders use full complexity RF chains (each antenna connected to an RF chain). One approach to design the unconstrained processors is by considering MMSE design to be proceeded in two steps. Firstly, the relays amplifying matrices are designed, then, given $\mathbf{G}_{\mathrm{u}}$ we design $\mathbf{W}_{\mathrm{u}}$ [17].

\section{A. Relay unconstrained processor $\mathbf{G}_{u, k}$ design}

The relay amplifying matrices $\left(\mathbf{G}_{u, k}\right)$ alongside the channels between the relays and the destinations $\mathbf{H}_{2, k}$ are used to cancel the effect of the channels between the source and the relays $\mathbf{H}_{1, k}$, and the noise vectors at the relays $\mathbf{n}_{1, k}$. Minimizing $\mathbb{E}\left\|s-\widetilde{\mathbf{G}_{\mathrm{u}}} \mathbf{y}_{r}\right\|$, where $\widetilde{\mathbf{G}_{\mathrm{u}}}=\left[\mathbf{H}_{2,1} \mathbf{G}_{u, 1}, \mathbf{H}_{2,2} \mathbf{G}_{u, 2}, \cdots, \mathbf{H}_{2, K} \mathbf{G}_{u, K}\right]$, and $\mathbf{y}_{r}=$ $\left[\mathbf{y}_{r, 1}, \mathbf{y}_{r, 2}, \cdots, \mathbf{y}_{r, K}\right]$. The MMSE solution is given by

$$
\begin{aligned}
\mathbf{G}_{u, k}= & \mathbf{H}_{2, k}^{H}\left(\mathbf{H}_{2, k} \mathbf{H}_{2, k}^{H}\right)^{-1}\left(\mathcal{R}_{s}^{-1}+\mathbf{H}_{1}^{H} \mathcal{R}_{\mathbf{n}_{1, k}}^{-1} \mathbf{H}_{1}\right) \\
& \mathbf{H}_{1, k}^{H} \mathcal{R}_{\mathbf{n}_{1, k}}
\end{aligned}
$$

B. Destination unconstrained processor $\mathbf{W}_{\mathrm{u}}$ design

Given $\widetilde{\mathbf{G}_{\mathrm{u}}}$, The MMSE solution for destination unconstrained processor could described as

$$
\begin{aligned}
\mathbf{W}_{\mathrm{u}}= & \left(\mathcal{R}_{s}^{-1}+\mathbf{H}_{1}^{H} \mathbf{G}_{\mathrm{u}}^{H} \mathbf{H}_{2}^{H} \mathcal{R}_{n_{e f f}}^{-1} \mathbf{H}_{2} \mathbf{G}_{\mathrm{u}} \mathbf{H}_{1}\right)^{-1} \mathbf{H}_{1}^{H} \\
& \mathbf{G}_{\mathrm{u}}^{H} \mathbf{H}_{2}^{H} \mathcal{R}_{n_{\text {eff }}}
\end{aligned}
$$

where

$$
\begin{gathered}
\mathcal{R}_{n_{e f f}}=\mathbf{H}_{2} \mathbf{G}_{\mathrm{u}} \mathcal{R}_{\mathbf{n}_{1}} \mathbf{G}_{\mathrm{u}}^{H} \mathbf{H}_{2}^{H}+\mathcal{R}_{\mathbf{n}_{2}} \\
\text { REFERENCES }
\end{gathered}
$$

[1] S. Rangan, T. Rappaport, and E. Erkip, "Millimeter-wave cellular wireless networks: Potentials and challenges," Proceedings of the IEEE, vol. 102, no. 3, pp. 366-385, March 2014.

[2] C. Jeong, J. Park, and H. Yu, "Random access in millimeter-wave beamforming cellular networks: issues and approaches," Communications Magazine, IEEE, vol. 53, no. 1, pp. 180-185, January 2015.

[3] C. Dehos, J. Gonzalez, A. De Domenico, D. Ktenas, and L. Dussopt "Millimeter-wave access and backhauling: the solution to the exponential data traffic increase in $5 \mathrm{~g}$ mobile communications systems?" Communications Magazine, IEEE, vol. 52, no. 9, pp. 88-95, September 2014.

[4] T. Rappaport, S. Sun, R. Mayzus, H. Zhao, Y. Azar, K. Wang, G. Wong, J. Schulz, M. Samimi, and F. Gutierrez, "Millimeter wave mobile communications for 5g cellular: It will work!" Access, IEEE, vol. 1, pp. 335-349, 2013.

[5] D. Lockie and D. Peck, "High-data-rate millimeter-wave radios," $\mathrm{Mi}$ crowave Magazine, IEEE, vol. 10, no. 5, pp. 75-83, August 2009.

[6] A. Sulyman, A. Nassar, M. Samimi, G. MacCartney, T. Rappaport, and A. Alsanie, "Radio propagation path loss models for $5 \mathrm{~g}$ cellular networks in the $28 \mathrm{ghz}$ and $38 \mathrm{ghz}$ millimeter-wave bands," Communications Magazine, IEEE, vol. 52, no. 9, pp. 78-86, September 2014.

[7] P. Wang, Y. Li, L. Song, and B. Vucetic, "Multi-gigabit millimeter wave wireless communications for 5G: from fixed access to cellular networks," Communications Magazine, IEEE, vol. 53, no. 1, pp. 168-178, January 2015.

[8] W. Roh, J.-Y. Seol, J. Park, B. Lee, J. Lee, Y. Kim, J. Cho, K. Cheun, and F. Aryanfar, "Millimeter-wave beamforming as an enabling technology for $5 \mathrm{~g}$ cellular communications: theoretical feasibility and prototype results," Communications Magazine, IEEE, vol. 52, no. 2, pp. 106-113, February 2014.

[9] S. Han, C.-L. I, Z. Xu, and C. Rowell, "Large-scale antenna systems with hybrid analog and digital beamforming for millimeter wave $5 \mathrm{~g}$," Communications Magazine, IEEE, vol. 53, no. 1, pp. 186-194, January 2015.

[10] O. El Ayach, S. Rajagopal, S. Abu-Surra, Z. Pi, and R. Heath, "Spatially sparse precoding in millimeter wave mimo systems," Wireless Communications, IEEE Transactions on, vol. 13, no. 3, pp. 1499-1513, March 2014.

[11] A. Alkhateeb, G. Leus, and R. Heath, "Limited feedback hybrid precoding for multi-user millimeter wave systems," Wireless Communications, IEEE Transactions on, vol. PP, no. 99, 2015.

[12] V. Venkateswaran and A.-J. van der Veen, "Analog beamforming in mimo communications with phase shift networks and online channel estimation," Signal Processing, IEEE Transactions on, vol. 58, no. 8 , pp. 4131-4143, Aug 2010. 
[13] J. Nsenga, A. Bourdoux, W. Van Thillo, V. Ramon, and F. Horlin, "Join $\mathrm{tx} / \mathrm{rx}$ analog linear transformation for maximizing the capacity at 60 ghz," in Communications (ICC), 2011 IEEE International Conference on, June 2011, pp. 1-5.

[14] O. Ayach, R. Heath, S. Abu-Surra, S. Rajagopal, and Z. Pi, "Low complexity precoding for large millimeter wave mimo systems," in Communications (ICC), 2012 IEEE International Conference on, June 2012, pp. 3724-3729.

[15] J. Tropp and S. Wright, "Computational methods for sparse solution of linear inverse problems," Proceedings of the IEEE, vol. 98, no. 6, pp. 948-958, June 2010.

[16] N. Khajehnouri and A. H. Sayed, "Distributed mmse relay strategies for wireless sensor networks," IEEE Transactions on Signal Processing, vol. 55, no. 7, pp. 3336-3348, July 2007.

[17] A. S. Behbahani, R. Merched, and A. M. Eltawil, "Optimizations of a mimo relay network," IEEE Transactions on Signal Processing, vol. 56, no. 10 , pp. 5062-5073, Oct 2008.

[18] J. Lee and Y. Lee, "Af relaying for millimeter wave communication systems with hybrid rf/baseband mimo processing," in Communications (ICC), 2014 IEEE International Conference on, June 2014, pp. 58385842 .
[19] A. Alkhateeb, O. El Ayach, G. Leus, and R. Heath, "Channel estimation and hybrid precoding for millimeter wave cellular systems," Selected Topics in Signal Processing, IEEE Journal of, vol. 8, no. 5, pp. 831846 , Oct 2014

[20] T. S. Rappaport, F. Gutierrez, E. Ben-Dor, J. N. Murdock, Y. Qiao, and J. I. Tamir, "Broadband millimeter-wave propagation measurements and models using adaptive-beam antennas for outdoor urban cellular communications," IEEE Transactions on Antennas and Propagation, vol. 61, no. 4, pp. 1850-1859, April 2013.

[21] M. Akdeniz, Y. Liu, M. Samimi, S. Sun, S. Rangan, T. Rappaport, and E. Erkip, "Millimeter wave channel modeling and cellular capacity evaluation," Selected Areas in Communications, IEEE Journal on, vol. 32, no. 6, pp. 1164-1179, June 2014.

[22] A. Maltsev, R. Maslennikov, A. Sevastyanov, A. Lomayev, and A. Khoryaev, "Statistical channel model for $60 \mathrm{ghz}$ wlan systems in conference room environment," in Proceedings of the Fourth European Conference on Antennas and Propagation, April 2010, pp. 1-5.

[23] M. Kim and Y. Lee, "Mse-based hybrid rf/baseband processing for millimeter-wave communication systems in mimo interference channels," Vehicular Technology, IEEE Transactions on, vol. 64, no. 6, pp. 2714-2720, June 2015 . 